\title{
Optimizing Efforts to Promote Mental Health on College and University Campuses: Recommendations to Facilitate Usage of Services, Resources, and Supports
}

\author{
Brett R. Harris, DrPH® \\ Brianna M. Maher, MPH \\ Leah Wentworth, PhD
}

\begin{abstract}
Mental health has long been a challenge on college and university campuses. Though it has historically taken a back seat to physical health, college administrators recently identified mental health as a key area for additional support. With the COVID-19 pandemic bringing mental health into the conversation across the country, there is no more critical and opportune time for colleges and universities to prioritize mental health. Many public and private universities alike have begun to do this, but how can they ensure that the services and resources they are providing and promoting get used and that students, faculty, and staff reap the benefits of these efforts? This commentary describes a set of universal steps the authors recommend based on their experience working with colleges and universities across New York State to increase uptake and usage of services.
\end{abstract}

The COVID-19 pandemic resulted in significant and prolonged negative impacts on the nation's mental health. College students were uniquely impacted by the pandemic, many being directed

Address correspondence to Brett R. Harris, University at Albany School of Public Health, One University Place, Rensselaer, NY, USA. bharris@albany.edu.

Address correspondence to Brianna M. Maher, University at Albany School of Public Health, One University Place,

Rensselaer, NY, USA. bharris@albany.edu.

Brett R. Harris, NORC at the University of Chicago, Bethesda, MD, USA.

Brianna M. Maher, NORC at the University of Chicago, Bethesda, MD, USA.

Leah Wentworth, National Alliance on Mental Illness, Arlington, VA, USA.

The Journal of Behavioral Health Services \& Research, 2022. 252-258. (c) 2021, National Council for Mental Wellbeing. DOI 10.1007/s11414-021-09780-2 
to immediately relocate from their college communities and transition to remote learning. ${ }^{1-3}$ Those mandated to relocate experienced more loneliness, grief, and generalized anxiety. ${ }^{3}$ In fact, Copeland and colleagues ${ }^{4}$ surveyed students prior to and following the onset of the pandemic and found negative effects on mood and behavioral and emotional functioning. Similarly, Charles and colleagues ${ }^{5}$ found greater mood disorder symptoms, perceived stress, and alcohol use.

Though the pandemic has had a significant impact on the mental health of college students, mental health was already an issue prior to the pandemic. In $2019,49.5 \%$ of college students reported loneliness, $35.7 \%$ depression, $22.1 \%$ depression that impaired academic performance, $31 \%$ anxiety, and $20.9 \%$ past 2 -week suicidal ideation. ${ }^{6}$ In addition, behaviors and experiences that significantly impact mental health-substance use, rape, and sexual assault-were also prevalent on college campuses. In fact, $26.4 \%$ of female and $6.8 \%$ of male undergraduates experienced rape or sexual assault by physical force, violence, or incapacitation. ${ }^{7}$ Another $38.3 \%$ reported past 2 -week binge drinking and $26.3 \%$ past month illicit substance use. ${ }^{6}$ Though colleges and universities are faced with competing demands and priorities, mental health and associated concerns are major and central issues for college students, not just in the aftermath of COVID-19 but for the long term.

There is no more critical and opportune time to prioritize mental health on college campuses, because the COVID-19 pandemic expanded the conversation around mental health and made it more acceptable to discuss. In fact, a recent national survey found that $52 \%$ of Americans reported being more open to talking about mental health as a result of COVID-19, and $81 \%$ reported that it is more important than ever to make suicide prevention a national priority, ${ }^{8}$ not just a priority for college campuses.

The efforts of the State University of New York (SUNY) 64-campus system provide a good example of taking action during the pandemic to increase mental health awareness, services, and supports. SUNY (1) partnered with Thriving Campus, a mobile-friendly app, to connect students to a network of over 6000 licensed mental health providers to break down help-seeking barriers; (2) added an additional tele-counseling hub; (3) expanded the University at Albany's Middle Earth peer-to-peer assistance hotline to all students across the 64-campus system; (4) launched a public awareness campaign to reduce stigma, increase knowledge of available services, and promote help-seeking; (5) partnered with the New York State College Health Association and the Suicide Prevention Center of New York to host a virtual suicide prevention symposium "Mental Health in the Changing Higher Education Landscape," free of charge to all students, faculty, and staff, which attracted over 520 participants; (6) made Question, Persuade, Refer (QPR) suicide prevention training free-of-charge; and (7) is promoting and tracking the use of Crisis Text Line. ${ }^{9}$

Mental health is serious and complex - ranging from emotional well-being to more serious conditions that may affect one's ability to function-and is connected with physical health, sleep patterns, and nutrition. Though colleges and universities can make policies, increase access to services and resources, and implement promising initiatives, it is more difficult to ensure that services and resources meet the needs of and get used by students impacted by stress in the environment, those with biological vulnerabilities, and those with serious mental illnesses. Though all campuses are unique, the following is a set of universal steps the authors recommend based on their experience working with colleges and universities across New York State to help change campus culture and increase uptake and usage of services. It is recommended that each campus test and evaluate any steps they choose to implement, as campuses cannot expect the mental health status of students to change without explicit planning and rigorous evaluation. 


\section{Start the Conversation on Mental Health and Increase Communication}

Though many students struggle with mental health and may be assessed and recommended for services, there is often a significant stigma that impedes help-seeking. Increasing the conversation around mental health is a first step to reducing stigma. ${ }^{10}$.

Communication with students should occur through a wide variety of channels to ensure as many individuals as possible receive the message from one of their preferred methods of communication. These may include university-wide emails, social media platforms, academic advisors, clubs, student leaders, professors, course curricula, resident assistants, coaches and trainers, and educational and social events. Communications may be most effective when incorporated into existing health and wellness campaigns using written, oral, and visual formats. The more students hear and see about mental health, the more it is normalized and the more they will talk to each other about it, seek out and complete training, help others, and seek help for themselves. The use of student testimonials and storytelling may help further reduce stigma and increase help-seeking as students relate best to their peers. Following increased mental health communications on the authors' campuses, students acknowledged the increase, expressed how much they were needed, and explained how receiving them made them feel their university was invested in their mental health.

\section{Make Mental Health Promotion and Suicide Prevention a Campus-Wide Effort}

The burden of mental health promotion and suicide prevention is often placed on college counseling centers. However, many students are not comfortable seeking help from formal counseling centers due to stigma, family and religious beliefs, cultural or racial/ethnic beliefs, a previous poor experience with formal counseling, inability to relate to therapists, and environmental factors. Therefore, it is important to engage all sectors of the university to ensure that every student is being reached and supported. This type of public health approach should include the health center, student body, faculty, staff, clubs, resident assistants, academic advisors, academic department heads, student affairs, and the larger community. Academic departments and their faculty and staff play a major role in student wellbeing and can influence students in positive ways, including help-seeking. Since mental health issues affect student learning, and there are many social determinants and contextual factors that impact mental health, academic departments should consider these issues as those of equality and inclusion among their student population.

Additionally, yet often overlooked, mental health promotion and suicide prevention efforts should target faculty and staff who are dealing with student mental health concerns ever more frequently. Not only do they need knowledge and skills to support their students, but they also need support for their own mental health. Training programs such as Mental Health First Aid can be a first step in helping to improve the self-awareness of mental health status. ${ }^{11}$ If faculty and staff are struggling with their own mental health, beliefs, or subsequent self-stigma, they may be less able to support their students and/or the mental health efforts of their university.

\section{Involve Students, Faculty, and Staff in the Development and Implementation of Mental Health Campaigns, Services, Resources, and Supports}

It is critical to include the thoughts, opinions, and experiences of students, faculty, and staff to ensure that services and resources meet their needs. This includes conducting surveys and holding focus groups, listening sessions, and forums on mental health and associated concerns such as 
substance use, rape, and sexual assault. Outreach should also target specific student populations to ensure that services and programs meet their needs-including undergraduates, graduate students, racial/ethnic minorities, veterans, LGBTQ + students, student-athletes, international students, and students on scholarship or financial aid, among others-as they all have unique college experiences and would benefit from tailored services and supports.

Once implemented, services and supports should be evaluated with feedback from their intended target population. This not only improves the appropriateness and acceptability of services but shows students, faculty, and staff that their college or university is invested in their mental health and well-being. Input and feedback should be used when promoting these services, resources, and supports. After conducting focus groups with students, the authors have regularly received gratitude for listening and ensuring students' voices are heard.

\section{Don't Just Make Training Available: Integrate It Into Academics and Student Life and Follow-up with Resources and Support}

Providing access to optional training will do little to train students, faculty, and staff in mental health and suicide prevention. Mass emails, continued outreach, and promotion on social media will only result in a small subset of the campus community completing training and acquiring the knowledge and skills that the administration hopes they will gain. Mental health or suicide prevention training should become a part of the set of annual trainings required for faculty and staff. These trainings could include Question, Persuade, Refer (QPR), Applied Suicide Intervention Skills Training (ASIST), and/or Kognito virtual simulations.

QPR is a one-hour training that instructs participants to recognize the signs of a suicidal crisis and how to question, persuade, and refer someone for help. Individuals trained in QPR reported increased knowledge and self-efficacy for interacting with suicidal individuals and were better able to engage in active listening, ask clarifying questions, and make appropriate referrals. ${ }^{12}$ ASIST is a 2-day interactive workshop in suicide first aid that teaches trainees to recognize when someone may be thinking about suicide and to create a plan that supports immediate safety. ${ }^{13}$ Research has shown that callers to a suicide hotline who spoke with ASIST-trained counselors were less depressed, overwhelmed, and suicidal compared to a control group. ${ }^{14}$.

Kognito's college-specific simulations teach students, faculty, and staff about psychological distress, how it could impact them, and what to do if they are concerned about others. A study of 41,510 students at 149 institutions of higher education and another study of 10,246 faculty and staff across 163 institutions of higher education found increases in total mental health skills, a 70\% increase in the number of students and a $47 \%$ increase in the number of faculty and staff discussing concerns with students, and a 53\% increase in the number of students and a $42 \%$ increase in the number of faculty and staff discussing referral options with students three months after completing the simulation. ${ }^{15,16}$.

To facilitate the completion of these or other trainings, trainings could become a part of required coursework, incentivized through extra credit or housing lotteries, or integrated into freshman and transfer student orientation. Going further, however, does a value or culture ever gain currency on campus without a course to support it? Creating a required course on mental health and imbedding suicide, rape, and sexual assault prevention training along with information about available services and supports not only ensures that students receive this information and gain critical knowledge and skills, but it also builds in the opportunity for follow-up and support so that they are equipped to use the knowledge and skills they gain. For example, Florida State University requires all freshman and transfer students to take trauma resilience training to help students adjust to campus, reduce stress, increase resilience, and improve mental health. ${ }^{17}$ Though promising, there is still a need to 
evaluate this strategy to assess its impact on college mental health. Last, emphasizing how everyone on campus has a role to play in preventing suicide, rape, and sexual assault will show the campus community how they all have a stake in mental well-being and will empower them to develop and hone these skills through training.

\section{Make Sure Information About Services and Supports Is Readily Available and Communicated to Students}

Though a campus may provide many services and supports, it is important that all students have access to information about key services at their fingertips. At minimum, this should include the number, website, and hours of the campus counseling center; the number of any campus peer support hotline; the National Suicide Prevention Lifeline and Crisis Text Line numbers, both hotlines which effectively lead to active rescues and connection to mental health services; ${ }^{18,19}$ and the National Helpline (for substance use) and the National Sexual Assault hotline numbers. Some campuses have included this critical information as a part of all course syllabi, while others have placed it on campus ID cards.

All of these resources have their unique place in the process of help-seeking. However, there is still a considerable amount of stigma attached to these critical resources and, when provided on their own or in a siloed campaign, students may view them as being only for someone in a "last resort crisis." Although these resources are ultimately for crisis situations, they are most effective when used before someone has made a life-threatening decision and is at the point of needing active rescue. So, what defines a "crisis," and how can institutions of higher education ensure that services meet the needs of all students who are experiencing a crisis? People with suicidal thoughts already feel like they are a burden to others, and this influences how they ask for help. In the authors' conversations with students who have suicidal thoughts, many expressed that they would not use services marketed as "crisis" services, because they do not feel that their problems amount to a crisis and would not want to take services from someone who may need them more. Changing the focus of messaging from one of crisis to one of hope and resilience and/or incorporating these resources into a larger campaign for health and wellness may help to reduce stigma and increase use.

\section{Assess the Needs of Students and Take a Staged Approach to Meet Their Needs}

Students' needs vary greatly. Some students struggle with mental health due to stressors in the environment, others' struggles are exacerbated by co-morbid alcohol or other drug use or by an incident of rape or sexual assault, and others may have a serious mental illness and/or biological vulnerabilities that have no precipitating event. Therefore, it is important to assess students' needs to understand the kinds of problems they may be experiencing and to more effectively route them to appropriate services, either on campus or by referral to community-based services.

Screening and assessment can be conducted for all students at the beginning of the fall semester via online survey; mandatory mental health checks by their primary care physician prior to arriving on campus or by the counseling center after arriving on campus; or any time during the semester by universal screening and assessment in college health and counseling centers. Assessments should be comprehensive and address issues commonly linked with mental health such as substance use, rape, and sexual assault. Following screening and assessment, college counseling centers or off-campus mental health service providers should determine a care plan based on level of risk, deliver services, and provide appropriate resources including information about local support groups and hotlines.

Screening, Brief Intervention, and Referral to Treatment (SBIRT) for substance use ${ }^{20}$ is an evidencebased model that has been tailored for use with college students (BASICS) ${ }^{21}$ and has more recently 
incorporated care for mental health concerns. ${ }^{22}$ Using this model, students receive a brief intervention at the college health or counseling center immediately following a positive screening. Those with significant concerns such as serious mental illness, a substance use disorder, or are a victim of rape or sexual assault receive a referral to specialty treatment providers, either in-house or to an external provider. For referrals to external providers, it is important to make a warm hand-off, engage in care coordination, and follow-up.

\section{Collaborate with Outside Organizations and the Community}

Colleges and universities have limited resources, only exacerbated by the COVID-19 pandemic, and lack of staff time and budgetary restraints often hinder mental health efforts. Active collaboration with external organizations and the outside community may help to alleviate this burden. Establishing a relationship with local mental health clinics can be beneficial in addressing future issues around care for students. Organizations like the American Foundation for Suicide Prevention (AFSP), the National Alliance on Mental Illness (NAMI), and the Rape, Abuse \& Incest National Network (RAINN) have local chapters or networks that can support colleges and universities. Other entities with which to collaborate include Active Minds, JED Campus, state and county-level mental health departments, student insurance companies or payers, local bar and restaurant associations, malls and shopping centers, and local police.

\section{Conclusion}

Making mental health a priority on college and university campuses requires a culture change, and implementing the steps described above may help to facilitate this change. The nation has started to see a change in the dialogue around mental health in the wake of the COVID-19 pandemic. Colleges and universities should take advantage of this and begin now, involving their campus community in development and implementation of their strategy. Researchers should study the impact of these implementation strategies on the mental health of college students, adding to the evidence base around these strategies. Only then will the services, resources, and supports provided be most fully utilized and the college or university see the positive outcomes they expect.

\section{Implications for Behavioral Health}

Provision of mental health services in college counseling centers is not sufficient to meet the ever-increasing mental health needs of college students. A multipronged, public health approach is required. This approach should include universal prevention efforts through messaging and classroom instruction; promotion of free mental health, substance use, and rape and sexual assault prevention resources; training of students, faculty, and staff in risk identification and referral; routine screening, assessment, and intervention; and provision of a wide array of easily accessible services both in and outside of college counseling centers. Buy-in, support, input, and participation of key stakeholders-including students and non-behavioral health professionals such as medical, public health, and student services staff-are critical to the success of this approach and should be sought early on in development. Plans for evaluation should be put into place prior to the implementation of any initiatives to ensure these efforts are having the intended impact on their student populations. 


\section{Declarations}

\section{Conflict of Interest The authors declare no competing interests.}

\section{References}

1. [1] Liu CH, Pinder-Amaker S, Hahm H, et al. Priorities for addressing the impact of COVID-19 pandemic on college student mental health. Journal of American College Health. 2020. DOI: https://doi.org/10.1080/07448481.2020.1803882.

2. [2] Son C, Hegde S, Smith A, et al. Effects of COVID-19 on college students' mental health in the United States: Interview survey study. Journal of Medical Internet Research. 2020;22(9):e21279. DOI: https://doi.org/10.2196/21279.

3. [3] Conrad RC, Hahm HC, Koire A, et al. College student mental health risks during the COVID-19 pandemic: Implications of campus relocation. Journal of Psychiatric Research. 2021;136:117-126. DOI: https://doi.org/10.1016/j.jpsychires.2021.01.054.

4. [4] Copeland WE, McGinnis E, Bai Y, et al. Impact of COVID-19 pandemic on college student mental health and wellness. Journal of the American Academy of Child and Adolescent Psychiatry. 2021;60(1):134-141. DOI: https://doi.org/10.1016/j.jaac.2020.08.466.

5. [5] Charles NE, Strong SJ, Burns LC, et al. Increased mood disorder symptoms, perceived stress, and alcohol use among college students during the COVID-19 pandemic. Psychiatry Research. 2021;296:113706. DOI: https://doi.org/10.1016/j.psychres.2021.113706.

6. Healthy Minds Network and the American College Health Association. The impact of COVID-19 on college student well-being. 2020. https:// www.acha.org/documents/ncha/Healthy_Minds_NCHA_COVID_Survey_Report_FINAL.pdf. Accessed October 30, 2020.

7. Cantor D, Fisher B, Chibnall S, et al. Report on the AAU campus climate survey on sexual assault and misconduct. 2020. https://www.aau. edu/sites/default/files/AAU-Files/Key-Issues/Campus-Safety/Revised\%20Aggregate\%20report\%20\%20and\%20appendices\%201-7_(01-162020_FINAL).pdf. Accessed November 5, 2021.

8. The Harris Poll. Public perception of mental health and suicide prevention survey results. August 2020. https://theactionalliance.org/sites/ default/files/suicide_and_mental_health_public_perception_survey_final_report_august_2020.pdf. Accessed October 30, 2020.

9. State University of New York (SUNY). SUNY launches a comprehensive plan to expand access to mental health services to every student. October 4, 2020. https://www.suny.edu/suny-news/press-releases/10-20/10-4-20/mental-health.html\#: :text=SUNY\%20Launches\%20a\% 20Comprehensive\%20Plan,Health\%20Services\%20to\%20Every\%20Student\&text=As\%20part\%20of\%20this\%20new,licensed\%20mental\% 20health\%20service\%20providers. Accessed November 16, 2020.

10. Niederkrotenthaler T, Reidenberg DJ, Till B, et al. Increasing help-seeking and referrals for individuals at risk for suicide by decreasing stigma: The role of mass media. American Journal of Preventive Medicine. 2014;47(3S2):S235-S243. DOI: https://doi.org/10.1016/j.amepre. 2014.06.010.

11. [11] Hung MSY, Lam SKK, Chow MCM. Nursing students' experiences of mental health first aid training: A qualitative descriptive study. Collegian. 2019;26(5):534-540. DOI: https://doi.org/10.1016/j.colegn.2019.02.006.

12. Quinnett P. QPR Gatekeeper Training for Suicide Prevention: The model, theory and research. National Registry for Evidence-based Programs and Practices (NREPP). http://nrepp.samhsa.gov/ViewIntervention.aspx?id=299. Published 2017. Accessed March 20, 2021.

13. LivingWorks Education. ASIST two-day training. https://www.livingworks.net/programs/asist/. Published 2016. Accessed March $20,2021$.

14. [14] Gould MS, Cross W, Pisani AR, et al. Impact of Applied Suicide Intervention Skills Training on the National Suicide Prevention Lifeline. Suicide Life Threatening Behaviors. 2013;43(6):676-691. DOI: https://doi.org/10.1111/sltb.12049.

15. Albright G, Goldman R, Shockley K. At-risk for university and college students: A longitudinal study with 41,510 students at 149 institutions. 2018. Kognito.

16. Albright G, Goldman R, Shockley K. At-risk faculty and staff: A longitudinal study with 10,246 faculty and staff members at 163 institutions. 2018. Kognito.

17. Antisa L. Building stronger students: FSU launches Student Resilience Project. Florida State University News. August 13, 2018. https:// news.fsu.edu/news/university-news/2018/08/13/building-stronger-students-fsu-launches-student-resilience-project/. Accessed November $16,2020$.

18. [18] Gould MS, Munfakh JLH, Kleinman M, et al. National suicide prevention lifeline: Enhancing mental health care for suicidal individuals and other people in crisis. Suicide and Life Threatening Behavirs. 2012;42(1):22-35. DOI: https://doi.org/10.1111/j.1943-278X.2011. 00068.x.

19. [19] Szlyk HS, Roth KB, Garcia-Perdomo V. Engagement with Crisis Text Line among subgroups of users who reported suicidality. Psychiatric Services. 2019;71(4):319-327. DOI: https://doi.org/10.1176/appi.ps.201900149.

20. Substance Abuse and Mental Health Services Administration (SAMHSA). Screening, Brief Intervention, and Referral to Treatment (SBIRT). 2021. https://www.samhsa.gov/sbirt. Accessed November 5, 2021.

21. Youth.gov. Brief Alcohol Screening and Intervention of College Students (BASICS). 2021. https://youth.gov/content/brief-alcohol-scree ning-and-intervention-college-students-basics. Accessed November 5, 2021.

22. [22] Sterling S, Kline-Simon AH, Weisner C, et al. Pediatrician and behavioral clinician-delivered screening, brief intervention and referral to treatment: Substance use and depression outcomes. Journal of Adolescent Health. 2018;62(4):390-396. doi:https://doi.org/10.1016/j.jadoh ealth.2017.10.016.

Publisher's Note Springer Nature remains neutral with regard to jurisdictional claims in published maps and institutional affiliations. 